\title{
Prevalencia de anticuerpos contra virus de influenza equina en equinos deportivos de la República Argentina durante 2015 y 2016
}

\author{
Prevalence of antibodies against equine influenza virus in sport horses \\ from Argentina during 2015 and 2016
}

\author{
Durante $E^{1}$, Marcos $A^{2^{*}}$, Ayerbe $M^{3}$, Chiricosta $A^{1}$, Segato $L^{1}$, Donato $M E^{2}$, \\ Capellino $\mathrm{F}^{2}$, D'Alessio $\mathrm{F}^{2}$, Piskorz $\mathrm{A}^{3}$, Carballo Longo $\mathrm{M}^{3}$ \\ ${ }^{1}$ Programa de Enfermedades de los Equinos, Dirección de Programación Sanitaria, SENASA; \\ 2Dirección de Epidemiología y Análisis de Riesgo, SENASA; ${ }^{3}$ Dirección de Laboratorio Animal, SENASA. \\ *Correo electrónico del autor: amarcos@senasa.gob.ar
}

\begin{abstract}
Resumen: Mediante un muestreo en dos etapas se analizó la población de equinos de deporte o recreación de toda la República Argentina que presenta una frecuencia regular (o alta) de movimientos y para los cuales la vacunación contra influenza equina es obligatoria. Mediante la técnica de inhibición de la hemaglutinación se evaluó la presencia de anticuerpos contra dos cepas del virus de la influenza equina: A/eq/2 La Plata 93 y A/eq/Argentina/E2345-1/2012. Los títulos obtenidos permitieron clasificar a los equinos en protegidos, parcialmente protegidos y no protegidos. El $57 \%$ y el $59 \%$ de los equinos fueron considerados como no protegidos contra la cepa La Plata 93 y Argentina 2012, respectivamente. El $34 \%$ fue considerado como parcialmente protegido contra la cepa La Plata 93 y el $33 \%$ contra la cepa Argentina 2012. Finalmente, el $9 \%$ y el $8 \%$ de los animales fueron considerados protegidos contra la cepa La Plata 93 y Argentina 2012, respectivamente. Se constató que los animales de mayor edad, con mayor cantidad de dosis de vacunas aplicadas y con la fecha de última vacunación más cercana a la fecha del muestreo, presentaron concentraciones mayores de anticuerpos. Menos del $10 \%$ de los equinos tuvo títulos protectores y un alto porcentaje títulos inferiores a lo requerido para evitar la enfermedad, lo cual implica que existe en Argentina una gran cantidad de equinos que pueden diseminar la enfermedad sin presentar sintomatología.
\end{abstract}

Palabras claves: influenza equina, vacunación, Argentina

\begin{abstract}
The population of sport equines of Argentina frequently transported was analyzed through a two-stage sampling. In this population vaccination against equine influenza is mandatory. Antibodies against two strains of equine influenza virus (A/eq/2La Plata 93 and A/eq/Argentina/E2345-1/2012) were evaluated through the hemagglutination inhibition technique. Based on the titer obtained, horses were classified as protected, partially protected and not protected. Fifty-seven per cent and $59 \%$ of the horses were not protected against La Plata 93 and Argentina 2012 strains, respectively. Thirtyfour per cent of the horses were partially protected against La Plata 93 and Argentina 2012 strains, respectively. Finally, $9 \%$ and $8 \%$ of the animals were protected against La Plata 93 and Argentina 2012 strains, respectively. Older animals with more vaccinations and with the last vaccination applied closer to the date of sampling had higher antibody levels. Less than $10 \%$ of the horses had protective titer and a high percentage had insufficient titer to avoid the disease, suggesting that in Argentina a large number of horses can spread the disease without showing clinical signs.
\end{abstract}

Key words: equine influenza, vaccination, Argentina 


\section{Introducción}

La influenza equina (IE) es una enfermedad respiratoria viral sumamente contagiosa. En los caballos el agente etiológico es el virus de influenza $A$, subtipos A equi 1 (H7N7) y equi 2 (H3N8). Los virus H7N7 se consideran extintos. Los virus H3N8 han evolucionado en dos linajes, linaje Americano y linaje Europeo. A su vez el linaje Americano evolucionó en tres sublinajes: Kentucky, Florida y Sudamericano. El linaje predominante es Florida, que se dividió en dos clados antigénicamente diferentes, Clado 1 y Clado 2 (Cullinane \& Newton, 2013). El sublinaje Sudamericano también se encuentra dividido en Clado 1 y Clado 2 (Olguin Perglione et al., 2016b).

Los brotes de IE suelen estar asociados a la concentración de los caballos en eventos ecuestres, donde adquieren la enfermedad. Luego la diseminan cuando son trasladados una vez terminado el evento ecuestre (van Maanen \& Cullinane, 2002). La medida profiláctica más importante es la aplicación de vacunas (Newton et al., 2000a).

En países endémicos las pérdidas económicas debidas a la enfermedad pueden minimizarse mediante la vacunación específica de caballos con traslados frecuentes (Gildea et al., 2010; Olguin Perglione et al., 2016a;).

La enfermedad puede manifestarse con fiebre, tos seca y áspera seguida de secreción nasal inicialmente serosa, que luego pasa a ser mucopurulenta. En animales vacunados, con inmunidad parcial, se observa que alguno de estos signos puede estar ausentes (Newton et al., 2006). Sin embargo, estos equinos pueden seguir excretando el virus y servir de fuente de infección para otros animales (Cullinane \& Newton, 2013).

En la República Argentina, en marzo de 1993, se produjo un brote de IE en los hipódromos de $\mathrm{Pa}$ lermo y de San Isidro, donde enfermaron aproximadamente el $40 \%$ de los equinos en entrenamiento. En octubre de 1994, otro episodio de intensidad menor afectó a los animales de dos años de edad. A fines de mayo de 1995, se notificó otro brote en el hipódromo de San Isidro, con la particularidad de haber afectado en forma variable a los equinos, lo que se atribuyó a los diferentes estados inmunitarios dentro de una población. En el periodo comprendido entre 2011 y 2016, en el Servicio Nacional de Sanidad y Calidad Agroalimentaria (SENASA) se recibieron 19 notificaciones de sospechas de influenza equina, con 12 eventos confirmados de la enfermedad y 238 equinos afectados. El último brote confirmado de IE en la Argentina se produjo en 2012, afectó principalmente a los hipódromos de la provincia de Buenos Aires (Azul, La Plata y San Isidro) y de la Ciudad Autónoma de Buenos Aires (Palermo) (Olguin Perglione et al., 2016a) y fue producido por un virus de influenza equina A subtipo A equi 2 (H3N8). Este mismo tipo de virus fue aislado, durante 2015, durante los brotes de influenza equina y casos individuales notificados en China, Francia, Alemania, Irlanda, Malasia, Suecia, Reino Unido y Estados Unidos (OIE, 2016).

En el período del estudio la aplicación de vacuna contra el virus de IE de manera previa a los movimientos de equinos era obligatoria, con excepción de aquellos movilizados con destino a faena. Las vacunas nacionales disponibles en el mercado contenían la cepa A/eq/La Plata/93 y las vacunas importadas contenían la cepa A/eq/Kentucky/1/97.

Dado que se desconoce la situación inmunológica real de la población de equinos de Argentina, el objetivo de este estudio fue estimar la prevalencia de anticuerpos contra IE en una subpoblación específica, como los equinos utilizados para deporte y recreación, y la magnitud de su respuesta humoral.

\section{Materiales y métodos \\ Información poblacional y marco de muestreo}

La población que se analizó fue la conformada por equinos de deporte o recreación de toda la República Argentina los que, de acuerdo a las actividades para las que son utilizados, presentan una frecuencia regular (o alta) de movimientos. En esta población la vacunación es obligatoria de forma previa a los movimientos (Resolución Ex SAGPyA N617/2005).

Sobre la base de los registros del Sistema Integrado de Gestión de Sanidad Animal (SIGSA) del Servicio Nacional de Sanidad y Calidad Agroalimentaria (SENASA), la población en estudio incluyó 1252 establecimientos y 92545 equinos.

Además, se realizó una encuesta para recabar los siguientes datos: edad de los equinos analizados, cantidad de vacunas aplicadas en dichos animales y fecha de aplicación de las vacunas.

\section{Diseño de muestreo}

Se realizó un muestreo probabilístico en dos etapas. La primera etapa consistió en la selección aleatoria de establecimientos proporcionalmente a su tamaño (cantidad de equinos por establecimiento) y, la segunda etapa, en la selección aleatoria simple de equinos dentro de cada establecimiento.

Los valores de los parámetros y supuestos propuestos se consignan a continuación.

- Prevalencia esperada: 60 \% de animales con anticuerpos contra el virus de IE.

- Tasa de homogeneidad (roh): dado que no se dispone de información previa se elige un nivel desconocido. 
- Cantidad de muestras que analizar por establecimiento: 15 . Surge sobre la base de aspectos biológicos, epidemiológicos, operativos y de recursos disponibles.

- Intervalo de confianza del $95 \%$, estándar para este tipo de estudios.

- Error relativo: se fija como valor aceptable de error relativo un $10 \%$.

De acuerdo con los parámetros y supuestos propuestos, el diseño de muestreo resultó en los valores consignados a continuación.

-Cantidad de animales a sangrar por establecimiento: 15 equinos.

-Establecimientos para analizar: 129.

-Cantidad de muestras totales: 1935.

La toma de muestras estuvo a cargo de los veterinarios locales del SENASA.

\section{Pruebas diagnósticas}

Se realizó el diagnóstico mediante la técnica de inhibición de la hemaglutinación (IHA) para dos cepas del virus de la IE subtipo 2 (H3N8): A/eq/La Plata/93 (linaje Americano, sublinaje Sudamericano, Clado 1) que fue aislada en la Facultad de Ciencias Veterinarias de la Plata en 1993, en adelante denominada LP93, y A/eq/Argentina/E2345-1/2012 (Linaje Americano, Sublinaje Florida, Clado 1) que fue aislada en el INTA Castelar en 2012 (Olguin Perglione et al., 2016a), en adelante denominada Argentina 2012. Este diagnóstico se realizó en el Laboratorio Animal del SENASA sobre la base del protocolo descripto por el Centro de Productos Biológicos Veterinarios del Departamento de Agricultura de Estados Unidos y según el manual de las pruebas de diagnóstico y de las vacunas para los animales terrestres de la OIE.

La cepa LP93 fue seleccionada para su análisis, dado que hasta 2014, fecha en la que los laboratorios comenzaron a actualizar las cepas de sus vacunas de IE, estaba contenida en ellas. La cepa Argentina 2012 fue seleccionada para su análisis por haber sido una de las cepas que en 2012 produjo un extenso brote de influenza equina en América del Sur.

\section{Clasificación de los animales}

Los animales se clasificaron considerando las siguientes categorías de protección sobre la base de los títulos de anticuerpos obtenidos:

- Protegidos (nivel de protección de infección y clínica): títulos $1 / 80$ y superiores.

- Parcialmente protegidos (nivel de protección clínica): títulos entre $1 / 20$ y $1 / 40$.
- No protegidos (susceptibles): título menor a $1 / 20$ o negativos.

Estas categorías se establecieron sobre la base de los títulos de anticuerpos, asumiendo una correlación entre cierto umbral de concentración de anticuerpos y su capacidad protectora.

\section{Análisis estadístico}

Los resultados de la encuesta fueron analizados a fin de evaluar posibles asociaciones entre la edad de los animales, la cantidad de vacunas aplicadas y la fecha de última vacunación con el título de anticuerpos. Los grupos se compararon en relación con la edad y la cantidad de vacunas recibidas a través del test no paramétrico de comparaciones de a pares KruskalWallis. Para comparar diferencias en los grupos con respecto a la fecha de la última vacunación se utilizó la prueba de Chi-cuadrado.

\section{Resultados}

Se muestrearon 120 establecimientos y 1677 equinos, obteniendo el siguiente porcentaje de cumplimiento del muestreo (Tabla 1).

Tabla 1. Cumplimiento de muestreo.

\begin{tabular}{lcc}
\hline & Establecimientos & Equinos \\
\hline Diseño & 129 & 1935 \\
Muestreados & 120 & 1677 \\
Cumplimiento (\%) & 93 & 87 \\
\hline
\end{tabular}

La prevalencia estimada para calcular la cantidad de establecimientos que muestrear fue del $60 \%$. Los resultados obtenidos arrojaron una prevalencia del $43 \%$ para la cepa LP93 (Tabla 2), que es la que presentó mayor cantidad de equinos con protección (protegidos y parcialmente protegidos).

Tabla 2. Prevalencia e intervalo de confianza para cepa LP93.

\begin{tabular}{lccc}
\hline $\begin{array}{l}\text { Prevalen- } \\
\text { cia }\end{array}$ & $\begin{array}{c}\text { IC } 95 \% \\
\text { inf }\end{array}$ & $\begin{array}{c}\text { IC 95 \% } \\
\text { sup }\end{array}$ & $\begin{array}{c}\text { Tasa de ho- } \\
\text { mogeneidad }\end{array}$ \\
\hline $43,23 \%$ & $37,23 \%$ & $49,24 \%$ & 0,42 \\
\hline
\end{tabular}

En la tabla 3 se detallan los resultados por cepa y categoría de protección.

A su vez, se observó que el $95 \%$ de los equinos muestreados presentaban exactamente los mismos títulos de anticuerpos para ambas cepas (Tabla 4).

En las figuras 1 y 2 se observa una cantidad superior de establecimientos con mayor proporción de animales sin protección, así como también que ambas cepas evaluadas presentan prácticamente la misma distribución de sus resultados. 
Tabla 3. Datos por cepa y categoría de protección

\begin{tabular}{lcccc}
\hline & Cepa LP93 & \multicolumn{3}{c}{ Cepa Argentina 2012 } \\
\hline $\begin{array}{l}\text { Categoría de } \\
\text { protección }\end{array}$ & $\begin{array}{c}\text { Cantidad } \\
\text { de equi- } \\
\text { nos }\end{array}$ & $\%$ & $\begin{array}{c}\text { Cantidad de } \\
\text { equinos }\end{array}$ & $\%$ \\
No protegidos & 952 & 57 & 990 & 59 \\
$\begin{array}{l}\text { Parcialmente } \\
\text { protegidos }\end{array}$ & 566 & 34 & 554 & 33 \\
Protegidos & 159 & 9 & 133 & 8 \\
\hline Total general & 1677 & 100 & 1677 & 100
\end{tabular}

Tabla 4. Detalle de resultados agrupados para ambas cepas.

\begin{tabular}{llr}
\hline Protección a las dos cepas & Cantidad de equinos & \% \\
\hline No protegidos & 951 & 57 \\
Parcialmente protegidos & 519 & 31 \\
Protegidos & 124 & 7 \\
Total con el mismo título & 1594 & 95 \\
\hline Total analizados & 1677 & 100 \\
\hline
\end{tabular}

Con respecto a las encuestas, solo 37 establecimientos (31\% de los muestreados) respondieron, por lo cual se pudieron analizar los datos correspondientes a 502 animales (30\% de los muestreados).

El promedio de edad de los equinos analizados fue de 6 años, con un rango entre 1 y 25 años.

A través del test de comparaciones de a pares Kruskal-Wallis se compararon los grupos y se observó que no hubo diferencias significativas de edad entre los equinos protegidos $(P)$ y los parcialmente protegidos (PP), pero sí de estos con respecto al grupo de los no protegidos (NP) (Tablas 5, 6, 7 y 8).

Tabla 5. Análisis estadístico de edad y categoría de protección para cepa LP93.

\begin{tabular}{llr}
\hline Ranking de medias & Tamaño de muestra & \\
\hline NP & 212,5 & 261 \\
PP & 285,8 & 178 \\
P & 316,1 & 63 \\
\hline Total & 251,5 & 502 \\
\hline Estadístico Kruskal-Wallis: $41,7068 . p=0,0000$
\end{tabular}

Figura 1: Categoría de protección desagregada por establecimiento y animal muestreado, cepa LP93.



Figura 2: Categoría de protección desagregada por establecimiento y animal muestreado, cepa Argentina 2012.

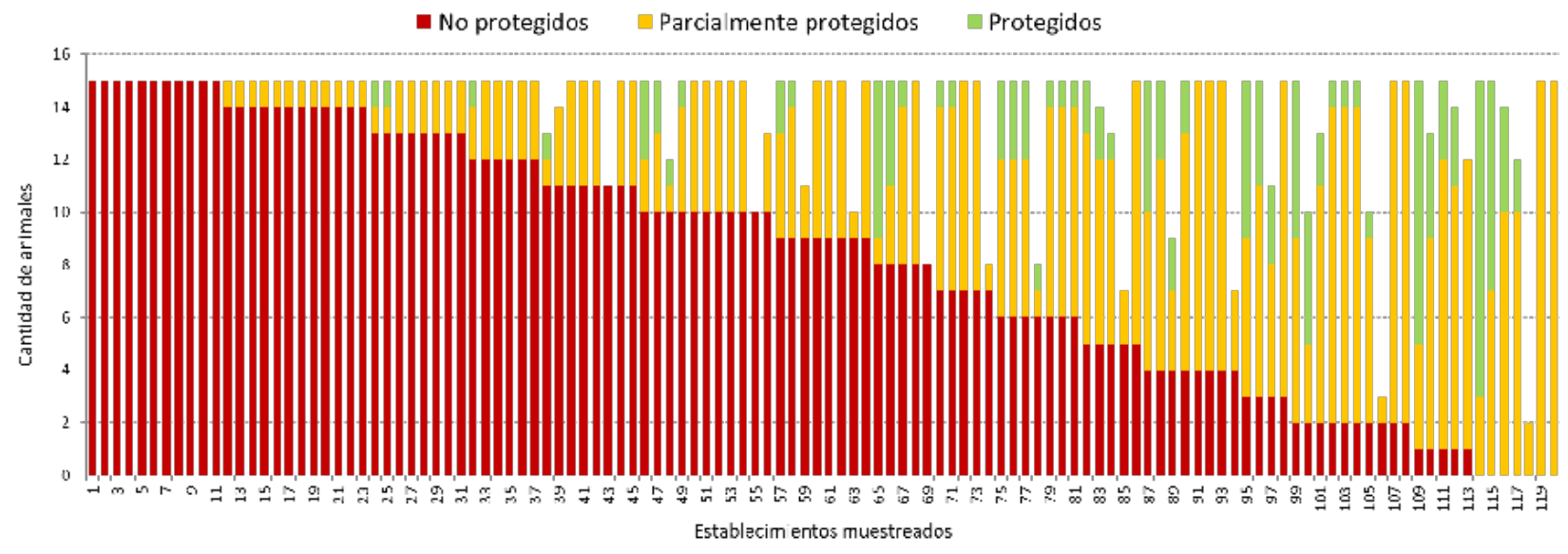


Tabla 6. Test de comparación de pares Kruskal-Wallis para edad y categoría de protección para cepa LP93.

\begin{tabular}{lcc}
\hline Media & Grupos homogéneos & \\
\hline PP & 316,11 & A \\
P & 285,79 & A \\
NP & 212,52 & B \\
\hline
\end{tabular}

Valor alfa: 0,05

Valor crítico de z: 2,394

Tabla 7. Análisis estadístico de edad y categoría de protección para cepa Argentina 2012.

\begin{tabular}{lcc}
\hline & Ranking de medias & Tamaño de muestra \\
\hline NP & 212,5 & 261 \\
PP & 285,8 & 178 \\
P & 316,1 & 63 \\
\hline Total & 351,5 & 502 \\
\hline
\end{tabular}

Estadístico Kruskal-Wallis: 41,7068. $p=0,0000$

Tabla 8. Test de comparación de pares Kruskal-Wallis para edad y categoría de protección para cepa Argentina 2012.

\begin{tabular}{lcc}
\hline & Media & Grupos homogéneos \\
\hline PP & 316,11 & A \\
P & 285,79 & A \\
NP & 212,52 & B \\
\hline
\end{tabular}

Valor alfa: 0,05

Valor crítico de z: 2,394

En la figura 3 se muestra que los animales del grupo NP fueron animales de menor edad (mediana: 4 años) que aquellos que pertenecieron a los grupos PP (mediana: 6,5 años) y P (mediana: 7 años). Estos resultados corresponden a la cepa LP93.

Muy similares resultados se obtuvieron cuando se analizaron los datos por edad entre los grupos NP (mediana: 4 años), PP (mediana: 7 años) y P (mediana: 7 años) respecto a la cepa Argentina 2012 (Figura 4).



Figura 3. Cantidad de animales distribuidos por edad y categoría de protección sobre la base de las encuestas realizadas. Cepa LP93.



Figura 4: Cantidad de animales distribuidos por edad y categoría de protección sobre la base de las encuestas realizadas. Cepa Argentina 2012.

El mismo análisis se repitió para detectar diferencias significativas entre los tres grupos mencionados y la cantidad de vacunas contra IE aplicadas. Para las dos cepas se detectaron diferencias significativas entre la media del grupo de equinos NP respecto a la media de los grupos P y PP (Tablas 9, 10, 11 y 12).

Tabla 9. Análisis estadístico de cantidad de vacunas aplicadas y categoría de protección para cepa LP93.

\begin{tabular}{lcr}
\hline Ranking de medias & Tamaño de muestra & \\
\hline NP & 182,7 & 242 \\
PP & 290,9 & 165 \\
P & 290,6 & 62 \\
\hline Total & 235 & 469 \\
\hline Estadístico Kruskal-Wallis: $75,9551$. & & \\
$p=0,0000$ & &
\end{tabular}

Tabla 10. Test de comparación de pares Kruskal-Wallis para cantidad de vacunas aplicadas y categoría de protección para cepa LP93.

\begin{tabular}{lcc}
\hline Media & Grupos homogéneos & \\
\hline PP & 290,86 & A \\
P & 290,57 & A \\
NP & 182,68 & B \\
\hline
\end{tabular}

Valor alfa: 0,05

Valor crítico de z: 2,394

Tabla 11. Análisis estadístico de cantidad de vacunas aplicadas y categoría de protección para cepa Argentina 2012.

\begin{tabular}{lcc}
\hline & Ranking de medias & Tamaño de muestra \\
\hline NP & 183,6 & 246 \\
PP & 290,9 & 169 \\
P & 294,2 & 54 \\
\hline Total & 235 & 469 \\
\hline Estadístico Kruskal-Wallis: $75,8686$. & \\
$p=0,0000$ &
\end{tabular}


Tabla 12. Test de comparación de pares Kruskal-Wallis para cantidad de vacunas aplicadas y categoría de protección para cepa Argentina 2012.

\begin{tabular}{lcc}
\hline & Media & Grupos homogéneos \\
\hline PP & 294,19 & A \\
P & 290,91 & A \\
NP & 183,60 & B \\
\hline
\end{tabular}

Valor alfa: 0,05

Valor crítico de z: 2,394

En las figuras 5 y 6 se observa que en el grupo NP (LP 93 mediana: 1,5 vacunas; Argentina 2012 mediana: 2 vacunas) se administraron menor cantidad de vacunas que en los grupos PP (LP 93 mediana: 4 vacunas; Argentina 2012 mediana: 4 vacunas) y $P$ (LP 93 mediana: 4 vacunas; Argentina 2012 mediana: 4 vacunas).

Se evaluó también el momento de la última vacunación con respecto al estado inmunitario de los equinos. Dividiendo a la población entre aquellos que según la encuesta recibieron la última vacuna antes del 2015 o directamente no fueron vacunados (grupo A) y los que fueron vacunados por última vez en 2015 (grupo B), se compararon ambos grupos mediante un test de Chi-cuadrado, detectándose diferencias significativas entre ambos grupos $(p<0,05)$. En el grupo A el muestreo detectó mayor cantidad de equinos NP que lo esperado y menos equinos $\mathrm{P}$ y PP de lo esperado (Tabla 13). En el grupo B se produjo lo opuesto, detectando el muestreo mayor cantidad de equinos $\mathrm{P}$ y PP que lo esperado y menor cantidad de equinos NP de lo esperado (Tabla 14).

\section{Discusión y conclusiones}

En un trabajo realizado en la región centro norte de la provincia de Santa Fe en 1999 se estudió la presencia de anticuerpos en una población de equinos de cría extensiva que no se encontraba vacunada contra influenza equina (Pinotti et.al.,

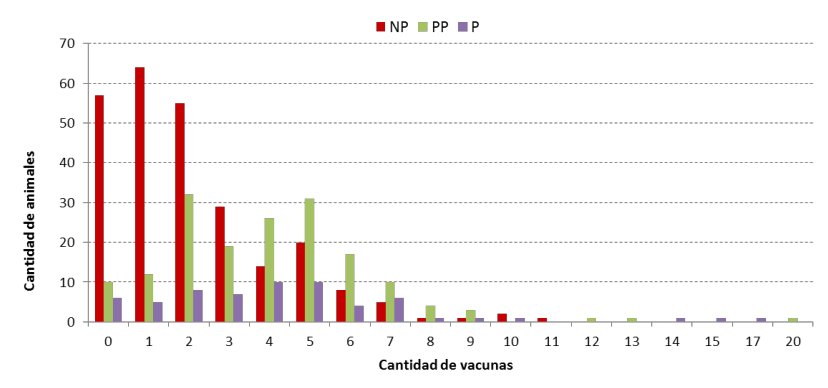

Figura 5. Cantidad de animales distribuidos por cantidad de vacunas aplicadas y categoría de protección sobre la base de las encuestas. Cepa LP93.
Tabla 13. Cantidad de animales distribuidos por última fecha de vacunación y categoría de protección sobre la base de las encuestas. Cepa LP93.

\begin{tabular}{lcccc}
\hline $\begin{array}{l}\text { Vacuna- } \\
\text { ción }\end{array}$ & PP + P & NP & Total parcial & $\begin{array}{c}\text { Total } \\
\text { general }\end{array}$ \\
\hline Grupo A & $10 \%$ & $25 \%$ & $35 \%$ & $100 \%$ \\
Grupo B & $39 \%$ & $26 \%$ & $65 \%$ & \\
\hline
\end{tabular}

Tabla 14. Cantidad de animales distribuidos por última fecha de vacunación y categoría de protección en base a las encuestas. Cepa Argentina 2012.

\begin{tabular}{lrrcc}
\hline $\begin{array}{l}\text { Vacuna- } \\
\text { ción }\end{array}$ & PP + P & NP & Total parcial & $\begin{array}{c}\text { Total } \\
\text { general }\end{array}$ \\
\hline Grupo A & $9 \%$ & $25 \%$ & $34 \%$ & $100 \%$ \\
Grupo B & $39 \%$ & $27 \%$ & $66 \%$ & \\
\hline
\end{tabular}

2002). El $23,74 \%$ de los equinos fueron positivos. Esto indica que la circulación del virus de IE en una población cerrada puede generar anticuerpos en un porcentaje un poco menor que los observados en el presente estudio.

Si bien se usaron distintos niveles de corte, los resultados de este estudio pueden compararse con los realizados por Carossino et al. (2014). La diferencia en la base de dilución entre ambos estudios se debe al tratamiento previo para la eliminación de posibles inhibidores inespecíficos de la hemaglutinación. En la mencionada investigación, el porcentaje de equinos protegidos y parcialmente protegidos en el hipódromo de Palermo fue de $97 \%$ y $74 \%$ en 2012 y 2013, respectivamente, mientras que para el hipódromo de San Isidro, los porcentajes fueron del $88,5 \%$ y $89 \%$ para los respectivos años. Estos valores resultan significativamente mayores a los obtenidos en nuestro trabajo. Esto puede deberse a la población analizada, ya que Carossino et al. (2014) estudiaron dos de los mayores hipódromos de Argentina, mientras que el muestreo presentado en esta publicación tuvo como población en estudio más de 1000 establecimientos en todo el país que poseían equinos de deporte o recreación.

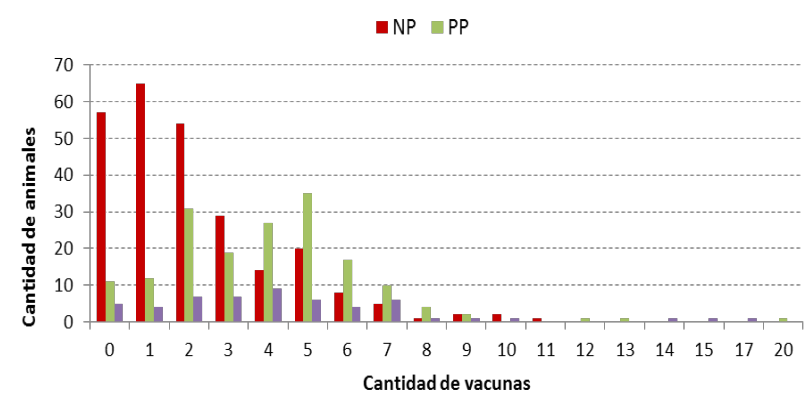

Figura 6. Cantidad de animales distribuidos por cantidad de vacunas aplicadas y categoría de protección sobre la base de las encuestas. Cepa Argentina 2012. 
Dos de las variables analizadas en la encuesta, cantidad de vacunas recibidas y fecha de la última vacunación, fueron identificadas como factores de riesgo asociados al nivel de títulos de anticuerpos contra IE en potrillos de raza Sangre Pura de Carrera (Newton et al., 2000b). En el modelo de regresión citado la asociación resulta positiva para cantidad de vacunas aplicadas previamente y negativa para fecha de última vacunación. Este modelo está en línea con los resultados de nuestro análisis estadístico, en el que los equinos NP tenían un promedio de vacunas aplicadas significativamente menor que los equinos $P$ y PP. Lo mismo ocurre respecto a la fecha de última vacunación en que el porcentaje de $\mathrm{P}$ y PP fue menor $(10 \%)$ en el grupo de equinos que habían recibido la última vacuna antes del 2015 o directamente no habían sido vacunados, que en el grupo vacunado por última vez en 2015 (P y PP: 39 \%).

Los resultados de la encuesta muestran que los animales protegidos y parcialmente protegidos son de mayor edad que los no protegidos. Esto podría explicarse por la mayor cantidad de vacunas recibidas a lo largo del tiempo. Se espera que estos equinos sean más resistentes a la infección. Sin embargo, está indicado en la bibliografía, que cuanto mayor es la cantidad de vacunas inactivadas aplicadas, se desarrollan más anticuerpos de reacción cruzada, que no mejorarían la calidad de la respuesta inmune (Newton et al., 2006). Por lo tanto, el mayor nivel de protección detectado en animales de mayor edad no sería garantía de una mejor protección inmunitaria.

Con este estudio se identificó que los equinos "protegidos", es decir, con nivel de protección suficiente para evitar la infección, representan el porcentaje más bajo, siendo del $9 \%$ para la cepa LP93 y del $8 \%$ para la cepa Argentina 2012. Esto indica que existe un bajo porcentaje de animales con niveles de inmunidad adecuados frente a esta enfermedad, ya sea de origen vacunal o por infección natural. Este valor asciende al sumar el grupo de los equinos "parcialmente protegidos"; sin embargo, el porcentaje continúa siendo más bajo que en los equinos que resultaron "no protegidos".

El $95 \%$ de los equinos muestreados presentaban los mismos niveles de anticuerpos para ambas cepas. Esto podría indicar que la vacuna brinda protección contra la cepa Argentina 2012. Sin embargo, vemos que los equinos no vacunados, vacunados con pocas dosis o vacunados por última vez antes del 2015, también tuvieron anticuerpos contra ambos antígenos analizados. Por lo tanto, no se puede definir si los anticuerpos son producto de la vacuna o de una infección con la cepa Argentina 2012. Es importante considerar que la cepa vacunal (LP 93) y la cepa de campo (Argentina 2012) se encuentran estrechamente relacionadas y comparten epitopes. Además, en 2014 la OIE emitió una recomendación de actualización de cepas vacunales de IE, por lo cual los equinos vacunados en 2015 podrían haber sido inmunizados con vacunas que contuviesen la cepa $\mathrm{A} / \mathrm{Eq} / 2 /$ Argentina/ E2345-1/2012. Esta actualización se realizó sobre la base de las Conclusiones y Recomendaciones emitidas por el Comité de expertos de la OIE en Influenza Equina en marzo de 2013 (OIE, 2013).

Se observó que los animales de mayor edad, con mayor cantidad de dosis de vacunas aplicadas y con la fecha de última vacunación más cercana a la fecha del muestreo, presentaron niveles de anticuerpos más altos, pero también existe mayor probabilidad de que hayan estado expuestos al virus de influenza equina a campo.

Sin embargo, menos del $10 \%$ de los equinos tuvieron títulos protectores. Por lo tanto, el $90 \%$ restante o no tuvo inmunidad (aproximadamente el $60 \%$ ) o tuvo una protección parcial (aproximadamente el $30 \%$ ). Esto último conlleva el riesgo de que los animales puedan diseminar la enfermedad sin presentar sintomatología.

Los resultados obtenidos no permiten concluir si el bajo porcentaje de animales con protección es debido a la vacuna misma, a la aplicación o al incumplimiento del cronograma de vacunación. Tampoco es posible diferenciar si la protección en aquellos que presentan inmunidad se debe a la vacunación o a la presencia de anticuerpos debido a una reacción por infección. Para poder realizar un diagnóstico de infección reciente, además de los signos clínicos, se deben obtener muestras pareadas con catorce días de intervalo entre ambas para constatar si hay conversión serológica (aumento en cuatro o más diluciones en el título); esto permitiría diferenciar si los anticuerpos son vacunales o debidos a una infección. Lo que queda claro es que hay un elevado porcentaje de animales que son "vírgenes" inmunológicamente a esta enfermedad y que son susceptibles ante a un posible ingreso del agente.

A partir de los resultados obtenidos, y con el objeto de disminuir la posibilidad de nuevos brotes de la enfermedad, se actualizaron las medidas de control obligatorias en Argentina. Se estableció que todo equino que permanezca o se movilice hacia un remate feria, exposición, establecimiento identificado como hipódromo, club hípico, stud, caballeriza, centro tradicionalista y/o a distintas actividades ecuestres que concentren equinos para trote, pato, polo, salto, doma, jineteada, prueba completa y endurance debe estar vacunado contra influenza equina, y dicha vacunación debe encontrarse vigente. La vigencia de la vacuna contra influenza equina es de tres, seis o más meses, según el tipo de vacuna utilizada, conforme al plazo que el SENASA otorgue en la aprobación de las 
distintas marcas y series, que estará determinado por la duración efectiva de la inmunidad demostrada en cada caso (Resolución SENASA N521/2016).

A futuro, sería adecuado diseñar un estudio que permita evaluar la efectividad de la vacunación y su cumplimiento, ya que los datos de vacunación se obtuvieron mediante encuestas en el momento de tomar las muestras.

\section{Agradecimientos}

Muy especialmente a los veterinarios y paratécnicos de las diferentes regiones del SENASA, ya que con su aporte y esfuerzo se logró el cumplimiento del presente trabajo.

\section{Conflicto de intereses}

No existe conflicto de intereses.

\section{Bibliografía}

Carossino M, Olguín Perglione C, Miguens Soubie A, Vissani A, Becerra L, Duffy SJ, Campodónico R, Arcuri C, Costa A, Zambruno T, Elgue F, Gómez MS, Zaba O, Barrandeguy ME. 2014. Estimación de los niveles de protección contra influenza equina en caballos en entrenamiento y competencia. La Especie Equina. 46:36-43.

Cullinane A, Newton JR. 2013. Equine influenza-A global perspective. Veterinary Microbiology. 167:205-14. doi: 10.1016/j.vetmic.2013.03.029.

Gildea S, Arkins S, Cullinane A. 2010. A comparative antibody study of the potential susceptibility of Thoroughbred and non-Thoroughbred horse populations in Ireland to equine influenza virus. Influenza and Other Respiratory Viruses. 4(6):363-72. doi: 10.1111/j.1750-2659.2010.00163.x.

Newton JR, Townsend HGG, Wood JLN, Sinclair R, Hannant D, Mumford JA. 2000. Immunity to equine influenza: relationship of vaccine induced antibody in young Thoroughbred race horses to protection against field infection with influenza A/equine-2viruses (H3N8). Equine Veterinary Journal. 32(1):65-74 doi: 10.2746/042516400777612116.

Newton JR, Lakhani KH, Wood JLN, Baker DJ. 2000. Risk factors for equine influenza serum antibody titres in young Thoroughbred racehorses given an inactivated vaccine. Preventive Veterinary Medicine. 46:129-41. doi: 10.1016/S0167-5877(00)00144-6.

Newton JR, Daly JM, Spenser L, Mumford JA. 2006. Description of the outbreak of equine influenza $(\mathrm{H} 3 \mathrm{~N} 8)$ in the United Kingdom in 2003 , during which recently vaccinated horses in Newmarket developed respiratory disease. Veterinary Record. 158:185-92 . doi: 10.1136/vr.158.6.185.

OIE. OIE Expert surveillance panel on equine influenza vaccine composition. 2013. http://www.oie.int/doc/ged/ D12875.PDF.

OIE. OIE Expert surveillance panel on equine influenza vaccine composition. 2016. http://www.oie.int/es/nuestraexperiencia-cientifica/informaciones-especificas-y-recomendaciones/gripe-equina/.
Olguin Perglione C, Gildea S, Rimondi A, Miño S, Vissani A, Carossino M, Cullinane A, Barrandeguy M. 2016. Epidemiological and virological findings during multiple outbreaks of equine influenza in South America in 2012. Influenza and Other Respiratory Viruses. 10(1):37-46. doi: $10.1111 /$ irv. 12349.

Olguin Perglione C, Golemba MD, Torres C, Barrandeguy M. 2016. Molecular epidemiology and spatio-temporal dynamics of the H3N8 equine influenza virus in South America. Pathogens. 5:61-74. doi: 10.3390/pathogens5040061.

Pinotti, M, Occhi, H, Lucca, E, Blainq, L, Luy, D, Gollan, A. 2002.Prevalencia de anticuerpos contra influenza equina en la región centro-norte de la provincia de Santa Fe, Argentina, durante los años 1997-99. Revista FAVE - Ciencias Veterinarias 1(2):26-31.

Resolución Ex SAGPyA N617/2005. Página web de Información Legislativa y Documental del Ministerio de Justicia y Derechos Humanos. http://servicios.infoleg.gob.ar/infoleglnternet/anexos/105000-09999/108872/norma.htm.

Resolución SENASA N521/2016. Página web de Información Legislativa y Documental del Ministerio de Justicia y Derechos Humanos. http://servicios.infoleg.gob.ar/infoleglnternet/anexos/265000-69999/265486/norma.htm.

van Maanen C, Cullinane A. 2002. Equine influenza virus infections: an update. Veterinary Quarterly. 24(2):79-94. doi: 10.1080/01652176.2002.9695127. 